\title{
Vortex Dipoles for Surface Quasigeostrophic Models
}

\author{
David J Muraki ${ }^{1}$ \\ Simon Fraser University, Burnaby BC, Canada \\ Chris Snyder \\ National Center for Atmospheric Research, Boulder $\mathrm{CO}^{2}$
}

May 15, 2003

\begin{abstract}
A new class of exact vortex dipole solutions is derived for surface quasigeostrophic (sQG) models. The analysis extends the two-dimensional barotropic modon to fully three-dimensional, continuously stratified flow. Dipole structures exist for a variety of sQG configurations. In particular, the basic dipole propagates counter to the phase speed of linear Rossby waves in the presence of uniform background gradients of the surface potential temperature or planetary vorticity, and reduces to the barotropic Lamb dipole in the limit of a thin sQG layer.
\end{abstract}

\footnotetext{
${ }^{1}$ Corresponding Author: David J Muraki, Department of Mathematics, Simon Fraser University, Burnaby BC, Canada, V5A 1S6. e-mail: muraki@math.sfu.ca

${ }^{2}$ The National Center for Atmospheric Research is sponsored by the National Science Foundation.
} 


\section{Introduction}

Steadily translating dipolar vortices, also known as modons, arise as exact solutions in many geophysical fluid systems (Flierl 1987). They can be viewed as a robust pair of oppositesigned vortices whose mutual interaction leads to steady propagation of the entire structure. Vortex dipoles have been used as models for cold-core oceanic rings (Flierl 1980), atmospheric blocks (McWilliams 1980, Butchart et.al. 1989), and tropopause troughs and jets (Hakim 2000, Cunningham and Keyser 2000). Such applications have been limited by the fact that, except for Berestov (1979), the available dipole solutions all come from barotropic or layered models. Coherent structures on the tropopause, in particular, are most naturally and simply described in surface quasigeostrophic (sQG) models (Held et al. 1995), which have been applied extensively to the midlatitude tropopause (Rivest et.al. 1992, Juckes 1994, Muraki and Hakim 2000, Hakim et al. 2002). In this paper, we add to the existing gallery of exact dipole solutions by deriving a three-dimensional, continuously stratified modon for sQG, together with several related variations to that solution.

\section{The Modon Construction \& Surface Quasigeostrophy}

The simplest sQG model consists of an atmosphere having infinite horizontal extent (in $x, y$ ), bounded below by a rigid lower surface $(z \geq 0)$, and under the assumptions of constant stratification. The dynamical equations of quasigeostrophy (QG) are advections involving the interior potential vorticity $(Q)$ and the surface potential temperature $\left(\Theta^{s}\right)$

$$
\begin{aligned}
& \frac{D Q}{D t}=Q_{t}+J(\Psi, Q)=0 \\
& \frac{D \Theta^{s}}{D t}=\Theta_{t}^{s}+J\left(\Psi^{s}, \Theta^{s}\right)=0
\end{aligned}
$$

where $J(f, g)=f_{x} g_{y}-g_{x} f_{y}$ is the horizontal Jacobian and superscript ${ }^{s}$ denotes surface $(z=0)$ quantities (Held, et.al. 1995). The horizontal winds $(U, V)$ and potential temperature 
$(\Theta)$ are given by geostrophy

$$
U=-\Psi_{y} \quad ; \quad V=\Psi_{x} \quad ; \quad \Theta=\Psi_{z}
$$

where the QG streamfunction $(\Psi)$, is obtained via the three-dimensional Poisson inversion

$$
\nabla^{2} \Psi=Q \quad ; \quad \Psi_{z}(x, y, 0)=\Theta^{s}
$$

with decay to uniform flow (specifically in the case of a dipole) imposed in all unbounded directions.

In the literature, the only steady dipole solutions for a three-dimensional, stratified fluid are related to the spherical QG modon of Berestov (1979). The usual starting point for a modon construction is the assumption that the potential vorticity (PV) and streamfunction satisfy one linear relation inside a region of trapped fluid, and different one outside. For the Berestov dipole the trapped region is a sphere of radius $R$, and the linear PV-streamfunction relation is

$$
Q=\left\{\begin{array}{ccc}
\alpha_{i} \Psi & \text { inside } & (0 \leq r<R) \\
\alpha_{o} \Psi & \text { outside } & (R<r<\infty)
\end{array}\right.
$$

where $r$ is the radial spherical coordinate $\left(r^{2}=x^{2}+y^{2}+z^{2}\right)$. The $\alpha$ coefficients are determined as part of the solution process. It is also required that the wind is uniform in the far-field. Details of this solution are also given in Flierl (1987). The essential property of the Berestov dipole is that its dynamics are associated entirely with interior PV.

Flows in $\mathrm{sQG}$, in contrast, have uniform PV and their dynamics are associated with a potential temperature disturbance on a horizontal boundary. Thus in sQG, the PV advection equation is satisfied identically by imposing uniform interior $\mathrm{PV}(Q=0)$. The dipole motion is then implied by the evolution of surface potential temperature (2). Our derivation begins by decomposing the streamfunction into a localized disturbance and a uniform zonal wind

$$
\Psi=\psi(r, \phi, z)+c r \sin \phi \quad ; \quad \Theta=\theta(r, \phi, z)=\psi_{z}(r, \phi, z)
$$


expressed in terms of cylindrical coordinates $\left(r^{2}=x^{2}+y^{2}\right)$. Here, a localized dipole is defined by the disturbance streamfunction $\psi$ which decays in all unbounded directions. Note that a stationary dipole in an easterly mean wind $\left(U^{\infty}=-c<0\right)$ would correspond, under a Galilean transformation, to a westward propagating dipole (having speed $c$ ) in a quiescent environment. The steady-state condition is obtained from the surface advection (2)

$$
J\left(\psi^{s}+\operatorname{cr} \sin \phi, \psi_{z}^{s}\right)=0
$$

The modon construction fulfills the above zero Jacobian condition by specifying the linear relation

$$
\psi_{z}^{s}=\left\{\begin{array}{ccc}
-\frac{\alpha}{R}\left(\psi^{s}+c r \sin \phi\right) & \text { inside } & (0 \leq r<R) \\
0 & \text { outside } & (R<r<\infty)
\end{array}\right.
$$

which imposes decay as $r \rightarrow \infty$ by setting the surface potential temperature exactly to zero outside the dipole boundary. The sQG dipole solution is thus obtained by solving the linear system that consists of the zero PV Laplace inversion

$$
\nabla^{2} \psi=0
$$

with the above modon relation (8) as a surface boundary condition, and far-field decay. There is an additional consistency relation requiring that the circle defining the surface-trapped fluid $(r=R)$ be itself a streamline of the flow - this determines the proportionality constant $\alpha$ and embodies the only nonlinear aspect of the solution method.

The inhomogeneous contribution in the dipole equation (8) has a $\sin \phi$ angular dependence. It follows that the streamfunction $\psi$ must inherit the same azimuthal structure. The general solution of the Laplace equation (9) for this mode has the integral representation

$$
\psi(r, \phi, z)=R c \sin \phi \int_{0}^{\infty} \hat{\psi}(\sigma) J_{1}(\sigma r / R) \exp (-\sigma z / R) \sigma d \sigma
$$

where $J_{1}(\cdot)$ is the Bessel function of order one, and $\hat{\psi}(\sigma)$ is a Fourier-Bessel coefficient to be determined from the surface boundary condition. The above integral is known as a Hankel 
transform and has many properties analogous to the Fourier integral, including an inverse transform (Morse and Feshbach 1953). Upon substitution of the integral representation (10) into the modon condition (8), application of the inverse transform obtains a linear integral equation for the coefficient $\hat{\psi}(\sigma)$. This derivation, details of which are deferred to Appendix A, results in the equation

$$
V(\sigma) \hat{\psi}(\sigma)=\alpha\left\{\int_{0}^{\infty} K(\bar{\sigma}, \sigma) \hat{\psi}(\bar{\sigma}) \bar{\sigma} d \bar{\sigma}+F(\sigma)\right\}
$$

which is a standard form known as a Fredholm integral equation of the second kind (Kondo 1991). The coefficient $V(\sigma)$, kernel $K(\bar{\sigma}, \sigma)$ and forcing $F(\sigma)$ are given by the expressions

$$
\begin{aligned}
V(\sigma) & =\sigma \\
K(\bar{\sigma}, \sigma) & =\int_{0}^{1} J_{1}(\bar{\sigma} s) J_{1}(\sigma s) s d s \\
F(\sigma) & =\int_{0}^{1} J_{1}(\sigma s) s^{2} d s
\end{aligned}
$$

where these integrals are evaluated exactly using standard Bessel identities (Appendix A). The coefficient $V(\sigma)$ will be the vehicle for generating the dipole variations of Section 3 .

Numerical solutions for $\hat{\psi}(\sigma)$ given $\alpha$ are simply obtained by matrix inversion after approximating the integral in (11) by a discrete quadrature (such as Simpson's rule) over a sufficiently large interval $(0 \leq \sigma \leq \Sigma)$. Radial profiles of the surface streamfunction (solid) and potential temperature (dashed) are shown by the dark curves in Figure 1a. The FourierBessel spectral amplitudes $\hat{\psi}(\sigma)$ are shown in Figure 1b (100 of 512 modes shown, $\Sigma=256$ ). The line indicates an asymptotic slope of $-7 / 2$ which is the characteristic (Fourier-Bessel) spectral decay expected when the $\theta$-field has a jump in the radial derivative.

The value $\alpha \approx 4.1213$ is found by a simple root-finding procedure that enforces the modon boundary $(r=R)$ to be a separating streamline $(\Psi=0)$. This feature of the solution is apparent in the total streamfunction $\Psi(x, y, 0)$ shown in Figure 2. Like other modons, the 
dipole's propagation speed is simply proportional to its amplitude. A positive value of $c$ makes the upper part of the dipole (Figure 2) a warm, cyclonic anomaly, which results in a modon that is a steady disturbance in an easterly incident wind. The maximum $u$ wind within these steady vortices is $\approx 7.76 \mathrm{c}$ and hence considerably faster than the incident wind. In time-dependent sQG computations, we have observed this dipole to be an extremely stable structure.

\section{Variations on a Theme}

\subsection{Asymmetric Dipole}

The basic sQG dipole of the previous Section is symmetric, and has no net surface vorticity. The modon of the barotropic vorticity equation allows a variation in which a superimposed monopole rider leads to an asymmetric dipole (Flierl et.al. 1983; also attributed to Bliss in Saffman 1992; Meleshko and van Heijst 1994). In the sQG case, there is also a dipole variant whose asymmetry causes a constant rotation of its velocity vector, and results in a circular trajectory (Figure 3). The motion of this asymmetric dipole is steady in a frame of reference in solid-body rotation about the center of the circular trajectory. Equivalently, we may consider the dipole to be embedded in a background flow in solid-body rotation about a point offset (by a distance $P$ ) from the dipole center, so that

$$
\begin{aligned}
\Psi & =\psi(x, y, z)-\frac{\omega}{2}\left(x^{2}+(y-P)^{2}\right) \\
& =\psi(r, \phi, z)+\omega P r \sin \phi-\frac{\omega}{2}\left(r^{2}+P^{2}\right)
\end{aligned}
$$

where $\omega$ gives the counter-clockwise angular frequency. With this background flow, the sQG Jacobian condition (7) becomes

$$
J\left(\psi^{s}+\omega P r \sin \phi-\omega \frac{r^{2}}{2}, \psi_{z}^{s}\right)=0
$$


yet leaves the Laplace inversion (9) intact. Note that the dropping of the constant $\omega P^{2}$-term from (13) does not affect the Jacobian. At this point, one could proceed to pose integral representations and derive corresponding integral equations as in the previous Section. However by invoking linearity and manipulating the basic dipole solution (10), the new streamfunction can be directly expressed using the same dipole coefficient $\hat{\psi}(\sigma)$

$$
\begin{aligned}
\psi(r, \phi, z) & =\omega P R \sin \phi \int_{0}^{\infty} \hat{\psi}(\sigma) J_{1}(\sigma r / R) \exp (-\sigma z / R) \sigma d \sigma \\
& +\omega R^{2} \int_{0}^{\infty} \hat{\psi}(\sigma) J_{0}(\sigma r / R) \exp (-\sigma z / R) d \sigma .
\end{aligned}
$$

The first integral is identical to that of the basic dipole (10) since the tangential velocity $\omega P$, as the equivalent linear speed, simply replaces $c$ in (7). The second integral of (15) arises from $r^{2}$-term in (14) and constitutes an axisymmetric (monopolar) contribution whose $r$-derivative is proportional to (10) by virtue of the Bessel identity $J_{0}{ }^{\prime}=-J_{1}$. This derivative relationship between the integrals of (15) mirrors the $r$ versus $r^{2}$-forcing terms in the Jacobian (14), and is also a feature of the barotropic vorticity dipole (Flierl et.al. 1983). The streamfunction and potential temperature profiles for the monopolar part is shown by the light curves in Figure 1a.

Contours of the full streamfunction $\Psi$ are shown in Figure 3 for a trajectory with radius $P=4 R$. For positive $\omega$, the large-scale flow is anti-cyclonic; equivalently, such a dipole will execute a counter-clockwise orbit in a quiescent environment. Note that the sQG axisymmetric rider is of single-signed vorticity; it differs significantly from the straight trajectory dipole-with-rider (Flierl et.al. 1981) which possesses no net vorticity and has been observed to be unstable (Swenson 1986b). In time-dependent computations, this sQG dipole variant has also been observed to be an extremely robust configuration. Such looping trajectories for dipoles have also been observed in computations of the barotropic vorticity system (Flierl et.al. 1983, Haupt et.al. 1993). Finally, note that the particular limit of zero frequency and large orbital radius $(\omega R \rightarrow 0$ and $\omega P \rightarrow c)$ recovers the simple travelling dipole. 


\subsection{Dipole in a Finite Layer}

The sQG formalism can be extended to a geometry that includes a zero potential temperature lid at height $H$. The integral representation for the Laplace solution (10) merely requires a change to the vertical profile

$$
\psi(r, \phi, z)=R c \sin \phi \int_{0}^{\infty} \hat{\psi}(\sigma) J_{1}(\sigma r / R) \frac{\cosh (\sigma(H-z) / R)}{\cosh (\sigma H / R)} \sigma d \sigma
$$

that ensures $\theta=\psi_{z}$ vanishes at $z=H$. The normalization in the denominator (16) is designed to recover exactly the basic dipole form (10) in the deep-layer limit $(H / R \rightarrow \infty)$. This change to the vertical mode structure introduces only a minor difference to the left-side of the integral equation (11) in the form of the coefficient

$$
V(\sigma)=\sigma \tanh (\sigma H / R)
$$

The proportionality constant $\alpha$ now becomes a function of the aspect ratio, $H / R$. This dependence is shown (dark curve) in Figure 4a.

In the limit of a very thin layer $(H / R \rightarrow 0)$, the lidded $\mathrm{SQG}$ dipole reduces exactly to the Lamb dipole solution of the barotropic vorticity equation (Lamb 1945, Meleshko etal. 1994). The (surface) disturbance streamfunction $\psi(r, \phi, 0)$ becomes

$$
\psi(r, \phi, 0) \rightarrow \begin{cases}R c \sin \phi\left(\frac{2}{z_{1}} \frac{J_{1}\left(z_{1} r / R\right)}{J_{0}\left(z_{1}\right)}-\frac{r}{R}\right) & \text { inside }(0 \leq r<R) \\ R c \sin \phi\left(-\frac{R}{r}\right) & \text { outside }(R<r<\infty)\end{cases}
$$

and $\alpha \sim\left(z_{1}\right)^{2} H / R$ (shown as the asymptote in Figure 4a) where $z_{1} \approx 3.8317$ is the first zero of the $J_{1}(\cdot)$ Bessel function. This illustrates the general principle that the largest horizontal scales (which are also deep) of this finite layer sQG dynamics behave roughly as those of the barotropic vorticity equation. Furthermore, as the layer thins, a smaller amplitude disturbance is sufficient to maintain the same dipole translation speed. This can be seen from the dependence of the normalized $\theta_{\max }^{s}$ on the aspect ratio, also shown (light curve) in 
Figure 4a. Lastly, the barotropic limit provides a useful benchmark on the accuracy of the numerical solver which has been verified against very small values of $H / R \ll 0.01$ without difficulty (not shown).

For this case, and all of the following dipole variants, the spatial structures differ only slightly from the basic dipole and are not shown. In addition, the asymmetric component of Section 3.1 can be also combined with this variation.

\subsection{Dipole in a Horizontal Temperature Gradient}

The effect of a background environment with a horizontal gradient of potential temperature can be incorporated without introducing interior PV. This is case with Eady shear, where a constant vertical shear of magnitude $\lambda$ is added to the full streamfunction to balance the constant horizontal gradient of potential temperature:

$$
\Psi=\psi(r, \phi, z)+(c-\lambda z) r \sin \phi \quad ; \quad \Theta=\psi_{z}(r, \phi, z)-\lambda r \sin \phi .
$$

This modifies the surface potential temperature condition

$$
J\left(\psi^{s}+c r \sin \phi, \psi_{z}^{s}-\lambda r \sin \phi\right)=0
$$

which admits the linear modon relation

$$
\psi_{z}^{s}+\frac{\lambda}{c} \psi=\left\{\begin{array}{cl}
-\frac{\alpha}{R}\left(\psi^{s}+c r \sin \phi\right) & \text { inside }(0 \leq r<R) \\
0 & \text { outside }(R<r<\infty) .
\end{array}\right.
$$

Using the same integral representation as (10), the change to the integral equation again involves the left-side coefficient

$$
V(\sigma)=\left(\sigma-\frac{\lambda}{c}\right)
$$

For positive shear and westerly flow $(\lambda>0, c<0)$, the left-side factor $V(\sigma)$ is strictly positive for $0 \leq \sigma<\infty$ and numerical solution of (11) proceeds uneventfully. The dependence of $\alpha$ and $\theta_{\max }^{s}$ against $\lambda / c<0$ is shown in Figure $4 \mathrm{~b}$. These dipoles in shear have been confirmed to evolve stably in time-dependent sQG simulations. 
With reversed (easterly) uniform flow $(\lambda>0, c>0)$, the factor $V(\sigma)$ is zero when $\sigma=\sigma_{c}=\lambda / c>0$ - the critical value where the dipole speed and vertical decay scale exactly match those of the family of edge waves with horizontal wavenumber $\sigma_{c} / R$. Attempts to solve the integral equation suggest that a singularity in the amplitude $\hat{\psi}(\sigma)$ coincides with this vanishing of $V(\sigma)$. We believe this to be an indication that a steady dipole does not exist, since such a localized structure (in a time-dependent setting) could possibly radiate through coupling with these edge waves. One connection that associates $\sigma_{c}$ with such a radiative process is the following exact, steady solution

$$
\psi(r, \phi, z)=J_{1}\left(\sigma_{c} r / R\right) \sin \phi \exp \left(-\sigma_{c} z / R\right)
$$

which also satisfies (21) in the far-field. The above solution has the undesirable property of having oscillatory tails in vorticity which have very slow $O(1 / \sqrt{r})$ radial decay. Such a standing wave solution corresponds to incoming Rossby waves from infinity which precisely conspire with their outgoing radiation to form a steady interference pattern.

\subsection{Dipole with Beta}

Although including the beta-effect introduces non-uniform PV, there is still a type of dipole whose dynamics are associated with surface potential temperature. Steady PV dynamics requires first that the Jacobian condition

$$
J\left(\psi+c r \sin \phi, \nabla^{2} \psi+\beta r \sin \phi\right)=0
$$

be satisfied in the interior. This is achieved by replacing the zero PV condition (9) with

$$
\nabla^{2} \psi-\frac{\beta}{c} \psi=0
$$

and inverted subject to the boundary condition (8). The general $\sin \phi$-mode solution for this interior condition is

$$
\psi(r, \phi, z)=R c \sin \phi \int_{0}^{\infty} \hat{\psi}(\sigma) J_{1}(\sigma r / R) \exp \left(-\sqrt{\sigma^{2}+\beta / c} z / R\right) \sigma d \sigma
$$


Modification to the integral equation (11) is again only required on the left-side

$$
V(\sigma)=\sqrt{\sigma^{2}+\beta / c}
$$

and, in contrast to the Eady shear case, the numerical solution now proceeds uneventfully for easterly flow $(\beta>0, c>0)$. The dependence of $\alpha$ and $\theta_{\max }^{s}$ on $\beta$ is shown in Figure 4c.

In analogous fashion to the case of Eady shear, a dipole in westerly flow $(\beta>0, c<0)$ introduces a change of sign in the square root at $\sigma_{c}=\sqrt{-\beta / c}$, and the modes below the cutoff $\left(\sigma<\sigma_{c}\right)$ no longer have upward decay. Here again, a manifestation of this singularity is a (barotropic) standing Rossby wave

$$
\psi(r, \phi, z)=J_{1}\left(\sigma_{c} r / R\right) \sin \phi
$$

that is isentropic everywhere and thus, trivially satisfies the far-field surface conditions. Note that the Eady shear and beta effects permit steady dipole propagation in directions opposite to that of linear Rossby waves. In addition, both solutions require a stronger disturbance than the basic dipole travelling at the same speed.

\section{Conclusions}

For models such as the barotropic vorticity equation, dipoles provide an exact prototype for a nonlinear, travelling coherent disturbance. The explicit construction of a three-dimensional dipole has been shown for a variety of surface quasigeostrophic configurations. As demonstrated for a lidded finite layer of decreasing height, the sQG dipole is a three-dimensional equivalent to the barotropic modon. Their comparative dynamics are hence quite similar, as the sQG dipoles are also found only to travel only in the direction opposite to Rossby wave phase velocities in the presence of Eady shear or Coriolis beta.

The list of variations presented here is by no means complete, and the construction technique allows likely extension to weak shear (Swenson, 1986a), tropopause deformation (Rivest et.al. 1992), and possibly, beyond QG corrections (Muraki et.al. 1999). This methodology 
provides a versatile family of three-dimensional steady disturbances for which we envision will find use in applications based upon sQG dynamics.

\section{Acknowledgements}

DJM gratefully acknowledges support through NSF DMR-9704724, NSERC RGPIN238928, the Alfred P. Sloan Foundation, and the Visitor Program of the Mesoscale and Microscale Meteorology Division at NCAR. We are indebted to Andreas Dörnbrack for alerting us to the paper of Meleshko and van Heijst. Finally, special thanks to Greg Hakim and Phil Cunningham for their field obs of sQG model dipoles and their enthusiastic support of this analytical work. 


\section{Appendix A}

The derivation of the integral equation (11) relies on the integral relations between $\psi(s)$ and its Hankel transform $\hat{\psi}(\sigma)$

$$
\begin{aligned}
& \psi(s)=\int_{0}^{\infty} \hat{\psi}(\sigma) J_{m}(\sigma s) \sigma d \sigma \\
& \hat{\psi}(\sigma)=\int_{0}^{\infty} \psi(s) J_{m}(\sigma s) s d s
\end{aligned}
$$

where $m$ is any non-negative integer index (Morse and Feshbach 1953). The second integral thus corresponds to the inversion formula that obtains the $\hat{\psi}(\sigma)$ coefficient for the integral representation of $\psi(s)$. Substitution of the integral representation (10) into the modon relation (8) at $z=0$ gives

$$
-\int_{0}^{\infty} \bar{\sigma} \hat{\psi}(\bar{\sigma}) J_{1}(\bar{\sigma} s) \bar{\sigma} d \bar{\sigma}=-R \alpha \mathcal{H}(1-s)\left\{\int_{0}^{\infty} \hat{\psi}(\bar{\sigma}) J_{1}(\bar{\sigma} s) \bar{\sigma} d \bar{\sigma}+s\right\}
$$

where $s=r / R$ and $\mathcal{H}(1-s)$ denotes the Heaviside step function which is zero for $s>1$. Note also that in (30) the (dummy) variable of integration has been replaced with $\bar{\sigma}$. Multiplying the above by $s J_{1}(\sigma s)$ and integrating from $s=0 \rightarrow \infty$ results in the integral equation (11). Note that the finite limits of the integrals for the kernel and forcing (12) are a consequence of the vanishing of the Heaviside factor for $s>1$ (after interchanging the order of the right-side integrals). The Bessel integrals for the kernel and forcing (12) are given explicitly by

$$
\begin{aligned}
& K(\bar{\sigma}, \sigma)= \begin{cases}\frac{\bar{\sigma} J_{1}(\sigma) J_{0}(\bar{\sigma})-\sigma J_{1}(\bar{\sigma}) J_{0}(\sigma)}{\sigma^{2}-\bar{\sigma}^{2}} & \text { for } \bar{\sigma} \neq \sigma \\
\frac{\left(J_{1}(\sigma)\right)^{2}-J_{0}(\sigma) J_{2}(\sigma)}{2} & \text { for } \bar{\sigma}=\sigma\end{cases} \\
& F(\sigma)=\frac{J_{2}(\sigma)}{\sigma} .
\end{aligned}
$$




\section{References}

Berestov, A.L., 1979: Solitary Rossby waves. Izv. Acad. Sci. USSR Atmos. Oceanic Phys., 15, 443-447.

Butchart, N., K. Haines and J.C.Marshall, 1989: A theoretical and diagnostic study of solitary waves and atmospheric blocking. J. Atmos. Sci., 46, 2063-2078.

Cunningham P. and D. Keyser, 2000: Analytical and numerical modelling of jetstreaks: Barotropic dynamics. Quart. J. Roy. Meteor. Soc., 126, 3187-3217.

Flierl, G.R., 1987: Isolated eddy models in geophysics. Ann. Rev. Fluid Mech., 19, 493-530.

Flierl, G.R., V.D. Larichev, J.C. McWilliams and G.M. Reznik, 1980: The dynamics of baroclinic and barotropic solitary eddies. Dyn. Atmos. Oceans, 5, 1-41.

Flierl, G.R., M.E. Stern and J.A. Whitehead, 1983: The physical significance of modons: Laboratory experiments and general integral constraints. Dyn. Atmos. Oceans, 7, 233-263.

Hakim, G.J., 2000: Climatology of coherent structures on the extratropical tropopause. Mon. Wea. Rev., 128, 385-406.

Hakim, G.J., C. Snyder, and D.J. Muraki, 2002: A new surface model for cyclone-anticyclone asymmetry. for cyclone-anticyclone asymmetry. J. Atmos. Sci., 59, 2405-2420.

Haupt, S.E, J.C. McWilliams and J.J. Tribbia, 1993: Modons in shear flow. J. Atmos. Sci., 50, 1181-1198.

Held, I.M., R.T. Pierrehumbert, S.T. Garner, and K.L. Swanson, 1995: Surface quasigeostrophic dynamics. J. Fluid Mech., 282, 1-20.

Juckes, M., 1994: Quasigeostrophic dynamics of the tropopause. J. Atmos. Sci., 51, 27562768.

Lamb, H., 1945: Hydrodynamics. Dover, 738 pp. 
Meleshko, V.V., and G.J.F. van Heijst, 1994: On Chaplygin's investigations of two-dimensional vortex structures in an inviscid fluid. J. Fluid Mech., 272, 157-182.

McWilliams, J.C., 1980: An application of equivalent modons to atmospheric blocking. Dyn. Atmos. Oceans, 5, 43-66.

Morse, P.M. and H. Feshbach, 1953: Methods of Theoretical Physics, Volume 1. McGrawHill, 997pp.

Muraki, D.J., C. Snyder and R. Rotunno, 1999: The next-order corrections to quasigeostrophic theory. J. Atmos. Sci., 56, 1547-1560.

Rivest, C., C.A. Davis and B.F. Farrell, 1992: Upper-tropospheric synoptic-scale waves. Part I: Maintenance as Eady normal modes. J. Atmos. Sci., 49, 2108-2119.

Saffman, P.G., 1992: Vortex Dynamics. Cambridge, 311pp.

Swenson, M., 1986a: Equivalent modons in simple shear. J. Atmos. Sci., 43, 3177-3185.

Swenson, M., 1986b: Instability of equivalent-barotropic riders. J. Phys. Oceanogr., 17, 492-506. 


\section{Figure Captions}

Figure 1: (a) Radial profiles of disturbance streamfunction and potential temperature for the basic dipole (dark) and the asymmetric monopole (light). The basic dipole streamfunction is scaled on $R c$, and the potential temperature on $c$. The monopolar streamfunction is scaled on $R^{2} \omega$, and the potential temperature on $R \omega$. (b) Log-log scatterplot of Fourier-Bessel amplitudes $\hat{\psi}(\sigma)$ as numerically computed from (11).

Figure 2: Contours of the full streamfunction for the basic dipole in a frame where the dipole is steady. The contour intervals are $R c / 2$. Positive values of $c$ correspond to an easterly incident wind.

Figure 3: Contours of the full streamfunction for the asymmetric dipole in a frame where the dipole is stationary. The contour intervals are $R \omega P$ where $P=4 R$. Positive values of $\omega$ correspond to an anticyclonic large-scale vortical flow.

Figure 4: Variation of the $\alpha$ parameter (dark) for each of the dipole variants: (a) finite layer, (b) shear, and (c) beta. The thin-layer $(H / R \rightarrow 0)$ asymptote is shown (thin dashed) for case (a). Also shown (light) is the variation of $\theta_{\max }^{s}$ normalized relative to the basic dipole of Section 2 $\left(\theta_{\max }^{s} \approx 9.24\right)$ 

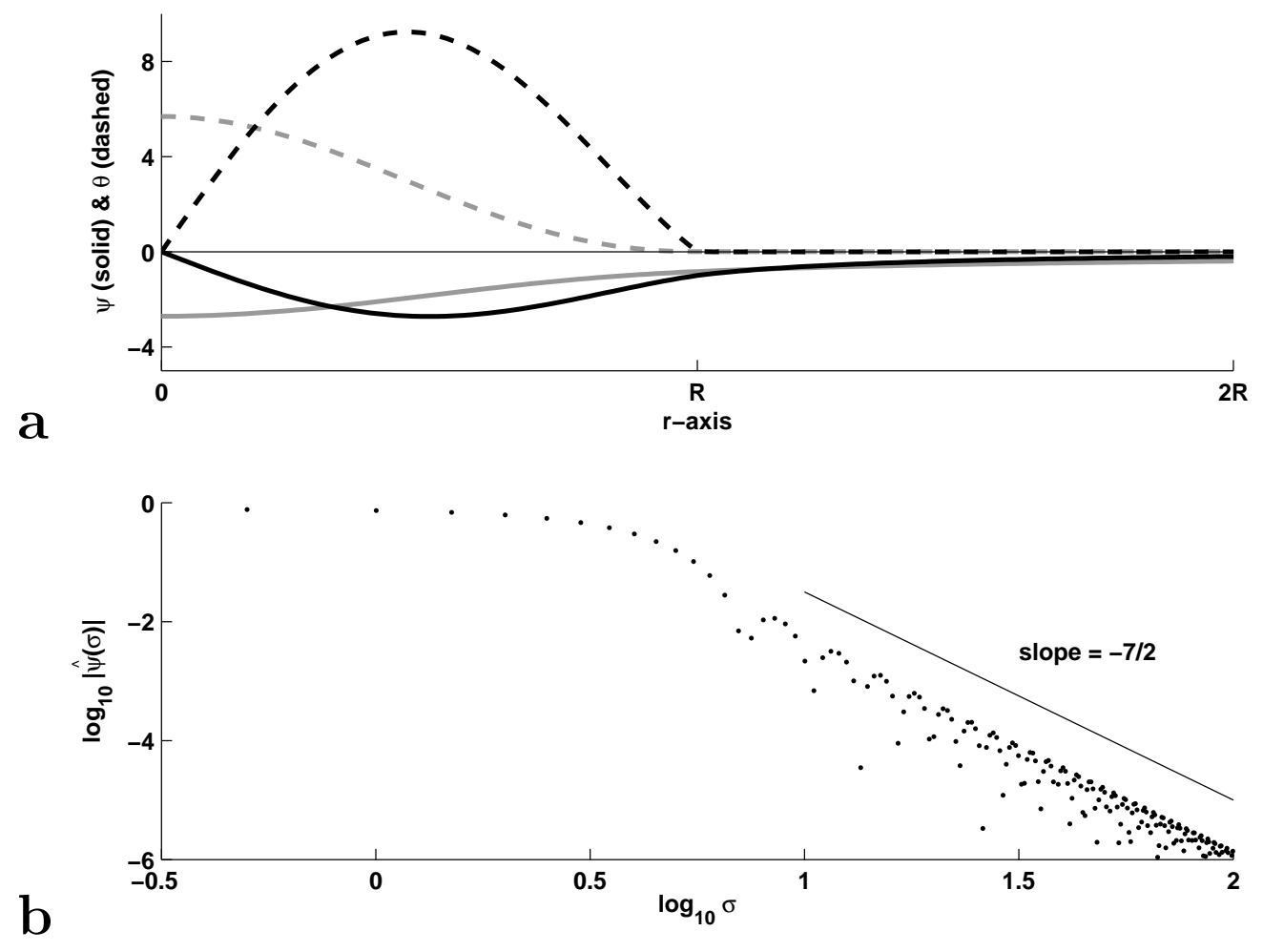

Figure 1: 


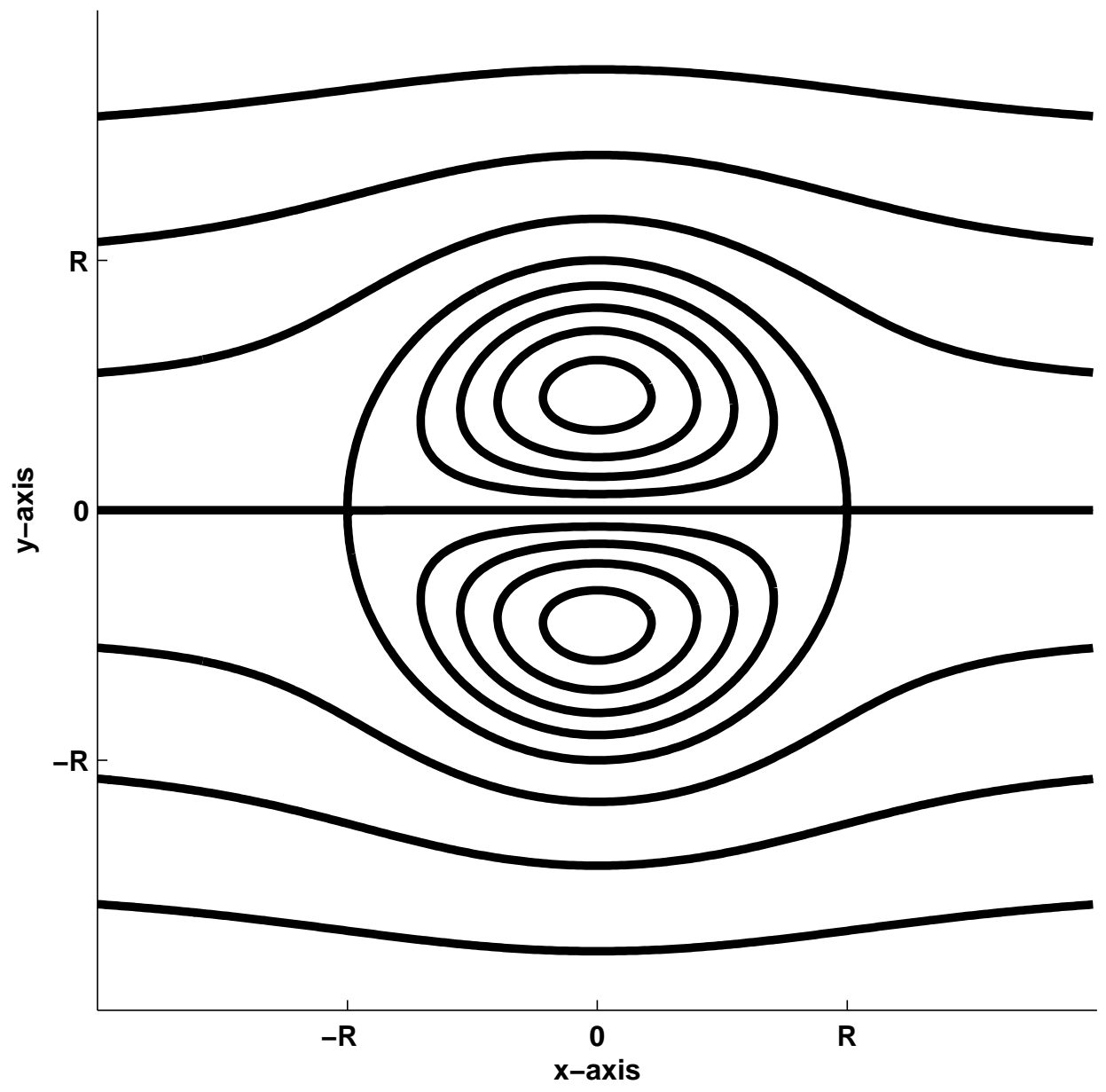

Figure 2: 


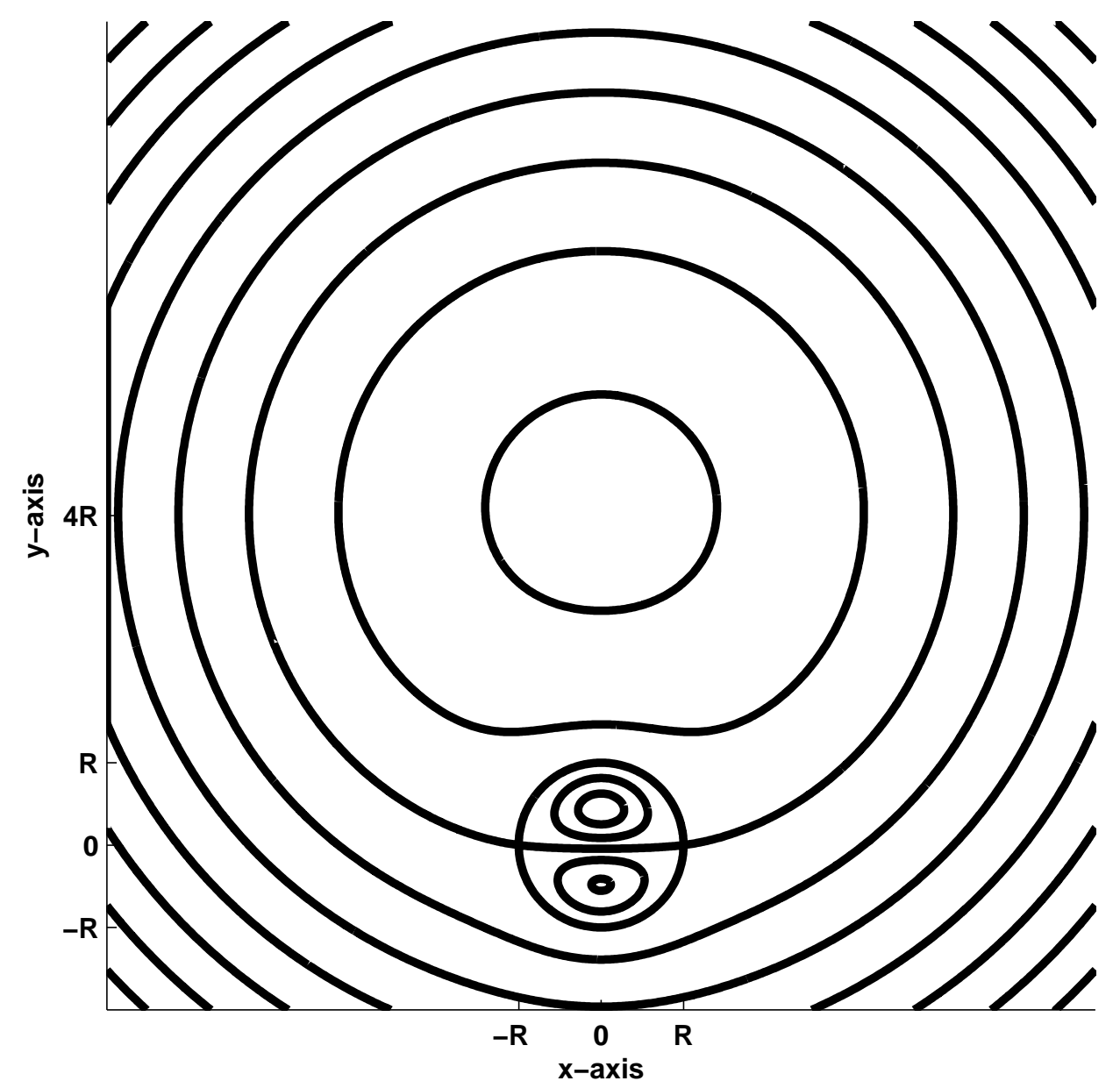

Figure 3: 


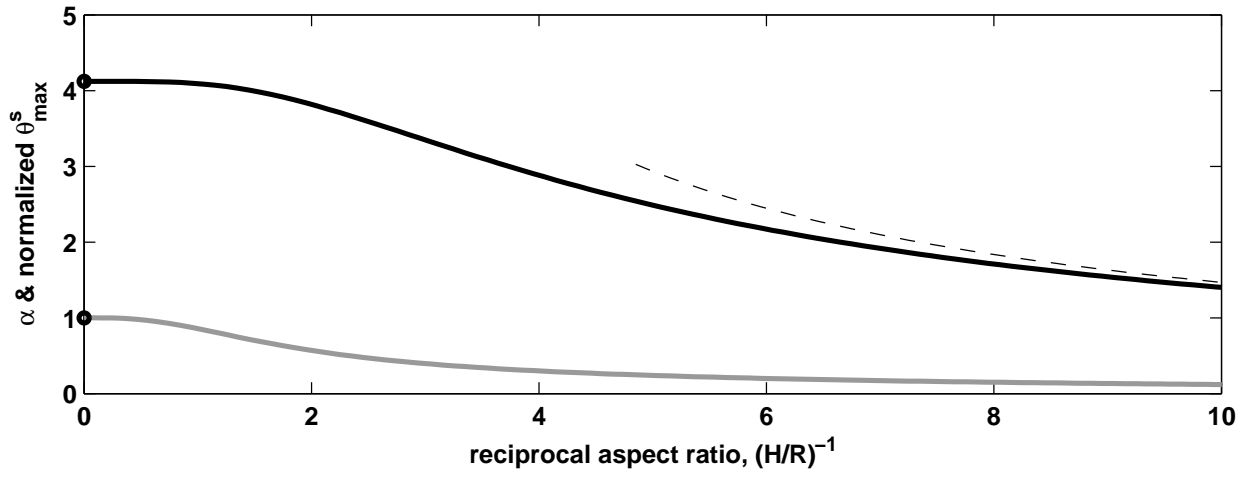

a
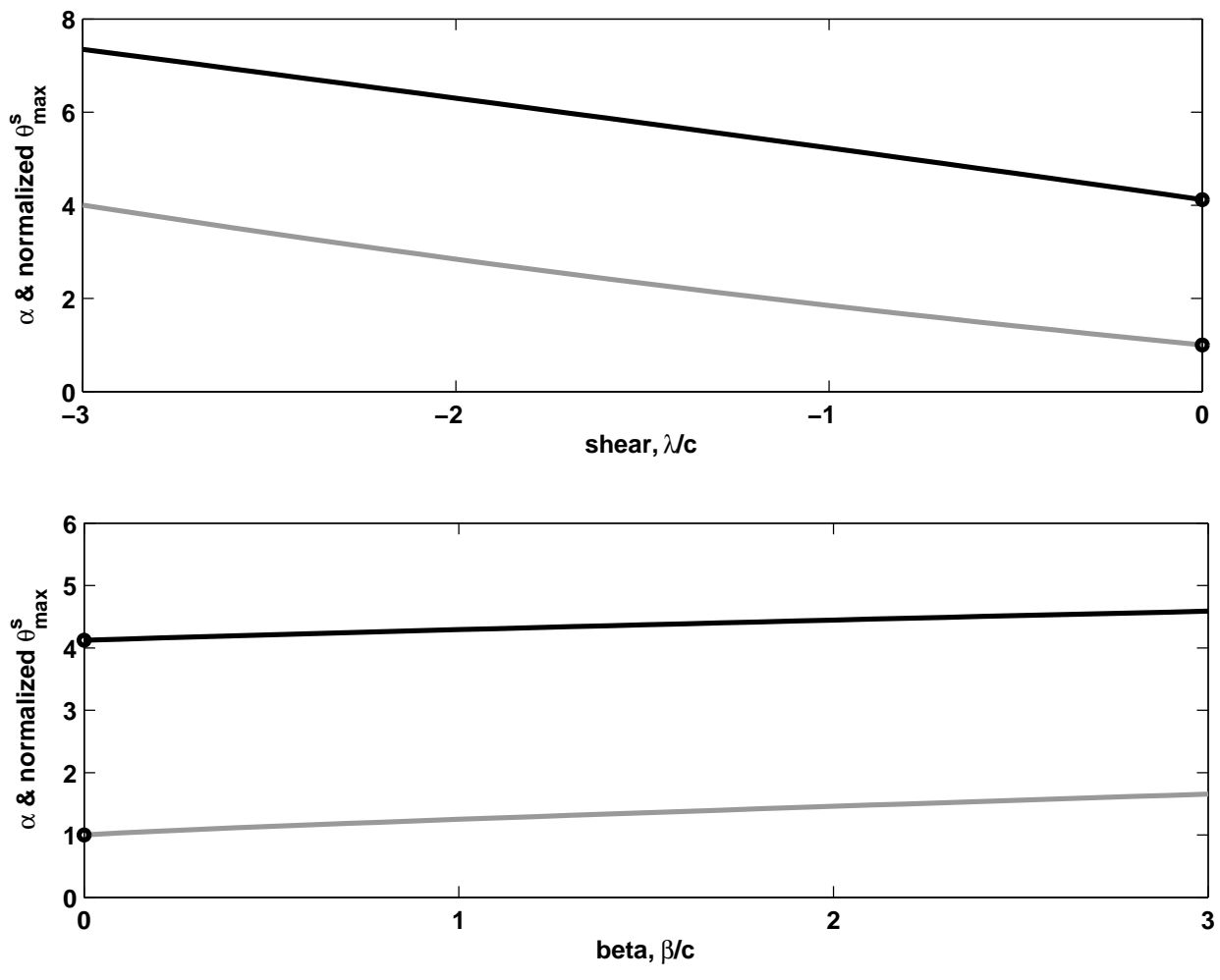

Figure 4: 